\title{
Development of a device for Field Emission Measurements on Carbon Nanotubes.
}

\section{Gabriel Z. Vitiello (IC), Fernando Alvarez (PQ), Luiz F. Zagonel(PQ).}

\begin{abstract}
In this work, we have developed a device for measurement of Field Emission of carbon nanotubes. The device is composed by a high vacuum chamber were an electrode is aproached to the carbon nanotube sample. A precisely controled voltage source generates an eletric field which is applied in the sample and the resulting current is measured. The implementation of a computer controlled piezo positioner, enabling 3D motion of the electrode, along with a computer controlled voltage source and ammeter will allow the device to become fully automated. This device will allow quicker and better caracterization of the filed emission properties of carbon nanotubes.
\end{abstract}

Key words: Field Emission, Carbon nanotubes, Instrumental development.

\section{Introduction}

Field Emission is a phenomenon observed when strong electric fields remove electrons from materials by tunnelling directly from its electronic bands. The intensity of the electric field can be drastically reduced by the use of materials with high aspect ratio, such as sharp needles. Carbon nanotubes present a very high aspect ratio and provide conductive and robust material to by applied as field emitters, which could have interesting technological applications. [1]

\section{Results and Discussion}

In this work, a device for measurement of Field Emission is being developed. The device integrates a vacuum chamber where an electrode, controlled via GPIB protocol in the software LabVIEW, is positioned close to a carbon nanotube sample for measurement. The voltage source and ammeter used is a Keithley Electrometer, model 6517A, which has a build in voltage source with sweep capabilities, a critical feature for automation. Also, it's low input bias current and high input impedance provides the accuracy and sensitivity needed for Field emission measurements. The electrode position will be computer controlled for automatic acquisition. All the positioners are from SmarAct of the SLC-24 series. These piezo positioners have high robustness and accuracy, allowing a travel range of hundreds of millimeters with a nanometric precision. In order to better understand and control the 3D motion, a prototype for 1D motion, in the $\mathrm{Z}$ direction, was proposed and produced as can been seen in Figure 1.

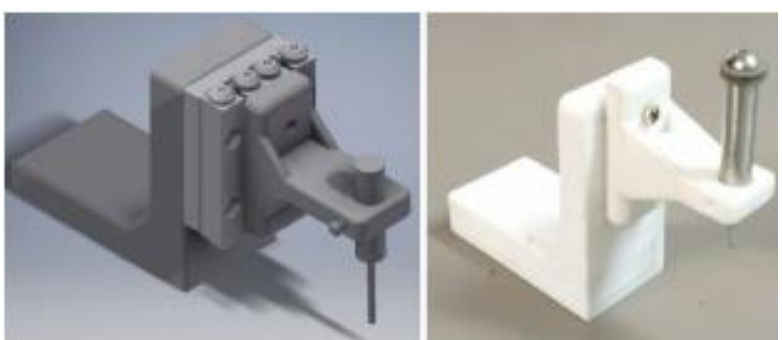

Figure 1: System prototype for measure automation in the $\mathrm{Z}$ direction. On the left, the preproduction, a CAD drawing of the prototype. And on the right, the $3 \mathrm{D}$ printed prototype without the piezo positioner.

\section{Conclusions}

Throughout all this project it was possible the development of instrumentation skills, or the use of instruments (build, measure and control) to monitor and control a process, the automated measurement of Field Emission. To this end, knowledge was gathered in the fields of high vacuum, sensitive electronics, part modeling and software control. The future prospects of this project are the implementation of the 3D motion as well as an improved graphic interface to better control the process.

\section{Acknowledgement}

This work is being supported by the grant \#2012/10127-5, São Paulo Research Foundation (FAPESP) and by the grant 480664/2013-7 $\mathrm{CNPq}$

${ }^{1}$ Walt A. de Heer, A. Châtelain, D. Ugarte, Science 1995, 270, 1179. DOI: $10.1126 /$ science.270.5239.1179 